\title{
Interaction between the human cytomegalovirus-encoded UL142 and cellular Snapin proteins
}

\author{
CHANG LIU, YING QI, YANPING MA, RONG HE, ZHENGRONG SUN, \\ YUJING HUANG, YAOHUA JI and QIANG RUAN
}

Virus Laboratory, The Affiliated Shengjing Hospital, China Medical University, Shenyang, Liaoning 110004, P.R. China

Received December 5, 2013; Accepted September 24, 2014

DOI: $10.3892 / \mathrm{mmr} .2014 .2829$

\begin{abstract}
Human cytomegalovirus (HCMV) infection can cause severe illness in immunocompromised and immunodeficient individuals. As a novel HCMV-encoded major histocompatibility complex class I-related molecule, the UL142-encoded protein (pUL142) is capable of suppressing natural killer (NK) cell recognition in the course of infection. However, no host factors that directly interact with HCMV pUL142 have been reported so far. In order to understand the interactions between HCMV pUL142 and host proteins, the current study used yeast two-hybrid screening, a GST pull-down assay and an immunofluorescence assay. A host protein, the SNARE-associated protein Snapin, was identified to directly interact and colocalize with HCMV pUL142 in transfected human embryonic kidney-293 cells. Snapin is abundantly expressed in the majority of cells and mediates the release of neurotransmitters through vesicular transport in the nervous system and vesicle fusion in non-neuronal cells. It is hypothesized that HCMV pUL142 may have an impact on the neurotransmitter release process and viral dissemination via interaction with Snapin.
\end{abstract}

\section{Introduction}

Human cytomegalovirus (HCMV) is a ubiquitous betaherpesvirus that has established a widespread and life-long latent infection in the majority of the global human population. HCMV infection is asymptomatic in healthy individuals, however, in immunosuppressed individuals, HCMV primary infection or reactivation usually causes serious side-effects,

Correspondence to: Professor Qiang Ruan or Dr Ying Qi, Virus Laboratory, The Affiliated Shengjing Hospital, China Medical University, 36 Sanhao Street, Shenyang, Liaoning 110004, P.R. China

E-mail: ruanq@sj-hospital.org

E-mail: qiy@sj-hospital.org

Key words: human cytomegalovirus, UL142 encoded protein, Snapin, interaction including HCMV pneumonia, hepatitis, encephalitis (1-3) and in certain cases, mortality.

HCMV has the largest genome of any characterized human virus, which is comprised of a DNA double helix of $>236 \mathrm{~kb}$ and 160 predicted open reading frames (ORFs), including unique long (UL) and unique short (US) regions (4-7). During extensive passage in vitro, a $15 \mathrm{~kb}$ sequence in the $\mathrm{UL} / \mathrm{b}$ ' region (UL133-UL151) was deleted from the widely used HCMV laboratory strain AD169 compared with the low passage Toledo strain (8). In recent studies, the UL/b' region has been demonstrated to be important for the dissemination, latency and virulence of HCMV in human hosts (9-12). The UL142 ORF, located within the $\mathrm{UL} / \mathrm{b}^{\prime}$ region, is $921 \mathrm{bp}$ in length and is predicted to encode a protein, presumably an important component in the inhibition of natural killer (NK) cell killing and the innate defense against HCMV (13).

It is well established that HCMV has developed effective approaches for hijacking and manipulating the host cellular processes to contribute to the viral replication and spread, primarily through interactions with host proteins. However, the identities of the host proteins that interact with the UL142 gene product (pUL142) in the infected cells remain unknown. The aim of the current study was to use a yeast two-hybrid screening system to identify cellular proteins that interact with pUL142 and to verify this interaction using a glutathione $\mathrm{S}$ transferase (GST) pull-down assay and then detect their co-localization in transfected embryonic kidney 293 (HEK-293) cells.

\section{Materials and methods}

Cells. HEK-293 cells (American Type Culture Collection, Manassas, VA, USA) were cultured in Dulbecco's modified Eagle's medium (HyClone, Logan, UT, USA), supplemented with $10 \%$ fetal bovine serum (HyClone), $100 \mathrm{U} / \mathrm{ml}$ penicillin and $100 \mathrm{mg} / \mathrm{ml}$ streptomycin (Boehringer-Ingelheim, Ingelheim am Rhein, Germany). The HEK-293 cells were maintained at $37^{\circ} \mathrm{C}$ in $5 \% \mathrm{CO}_{2}$.

Yeast two-hybrid screening. A yeast two-hybrid screening system (Matchmaker GAL4 Two-Hybrid System 3; Clontech Laboratories, Inc., Mountainview, CA, USA) was used to identify pUL142 interacting proteins from a human fetal brain cDNA library (pACT2-cDNA; Clontech Laboratories, Inc.). The full ORF sequence of the UL142 gene was amplified by 
polymerase chain reaction (PCR) using HCMV H strain DNA (GenBank no. GQ981646; Shenyang, China), and inserted into the BamHI sites of a pGBKT7 vector (Clontech Laboratories, Inc.) generating a plasmid (pGBKT7-UL142) that expresses a UL142 fusion protein with the binding domain (BD) to be used as a bait plasmid for the two-hybrid screening. The constructed plasmid was confirmed by DNA sequencing using the dideoxy chain-termination method carried out by the Beijing branch of the Invitrogen Life Technologies company (Invitrogen Life Technologies, Carlsbad, CA, USA). The pGBKT7-UL142 and pACT2-cDNA plasmids were sequentially transformed into the AH109 yeast host strain (Clontech Laboratories, Inc.) by electroporation (Bio-Rad, Hercules, CA, USA) according to the protocol provided by the manufacturer. The transformants were selected by seeding the yeast onto plates containing X-a-Gal (\#8061-1, Clontech Laboratories, Inc) and minimal synthetic dropout (SD) medium (Clontech Laboratories, Inc) lacking adenine (Ade), histidine (His), tryptophan (Trp) and leucine (Leu), and incubated for 3-7 days at $30^{\circ} \mathrm{C}$. According to the chromogenic reaction of $\alpha$-galactosidase activity, colonies that turned blue were retained and the positive results were confirmed by repeat assays. Inserts of the selected clones were sequenced by the dideoxy chain-termination method and analyzed by the Blast network service at the National Center for Biotechnology Information (http://www.ncbi.nlm. gov/blast).

In vitro translation reactions. Biotinylated pUL142 was expressed using the pGBKT7-UL142 plasmids $(1 \mu \mathrm{g})$ and a TNT T7 Quick Coupled Transcription/Translation system (Promega, Madison, WI, USA) according to the manufacturer's instructions. A $2-\mu 1$ aliquot of the biotin-containing translation products was separated directly on a sodium dodecyl sulfate-polyacrylamide (SDS-PAGE) gel (10\%) and transferred onto a $0.2 \mu \mathrm{m}$ polyvinylidene difluoride (PVDF) membrane (Millipore, Temecula, CA, USA). The biotinylated pUL142 $(55 \mathrm{kDa})$ that reacted to streptavidin-horseradish peroxidase (streptavidin-HRP, 1:10,000; Promega) was visualized using an enhanced chemiluminescence western blotting detection system (Bio-Rad).

GST pull-down assay. The Snapin sequence of the pACT2-Snapin, which was identified by yeast two-hybrid screening, was obtained by digestion with restriction endonucleases EcoRI and XhoI (Takara Bio, Inc., Dalian, China), and inserted into the GST-tagged pGEX-4T-2 vector (Pharmacia Biotech, Inc., Piscataway, NJ, USA), designated as the GST-Snapin fusion protein expression plasmid. The GST pull-down experiment was performed according to the manufacturer's instructions (MagneGST ${ }^{\mathrm{TM}}$ Pull-Down System; Promega). The GST-Snapin fusion proteins (43 kDa) were expressed and extracted from the BL21 Escherichia coli strain (Tiangen, Beijing, China) transfected with the GST-Snapin fusion protein expression plasmid. GST-Snapin fusion proteins $(200 \mu \mathrm{l})$ were incubated with MagneGST ${ }^{\mathrm{TM}}$ particles, which allow for the capture of a GST-labeled protein and protein complex, for $30 \mathrm{~min}$ at room temperature on a rotating platform. Following three washes of GST-Snapin fusion proteins, the particles were resuspended in $20 \mu \mathrm{l}$ GST binding/wash buffer. Biotinylated UL142 proteins $(80 \mu \mathrm{l})$ were added to the resuspended particles to a final volume of $800 \mu \mathrm{l}$ GST binding/wash buffer. Following incubation at $4^{\circ} \mathrm{C}$ for $1.5 \mathrm{~h}$ on a rotating platform, the reaction mixture was recovered by a magnetic stand (Promega). The prepared products were analyzed, using western blotting to detect the GST-Snapin using a mouse anti-GST monoclonal antibody (Pierce, Logan, UT, USA), and detecting the captured biotinylated UL142 proteins as mentioned above.

Immunofluorescence assay. The full-length UL142 coding sequence was obtained by PCR from HCMV H strain DNA. EcoRI and KpnI sites (underlined) were incorporated into the 5 ends of the amplicon with the following primers: forward, 5'-CCGGAATTCACGGAT TGA ATGGGC GTGTT-3', and reverse 5'-CGGGGTACCTTACTGACC GCGCCATAC-3', respectively. The PCR products were inserted into the mammalian expression vector enhanced green fluorescent protein (GFP) plasmid (pEGFP-N1) (BD Biosciences, Franklin Lakes, NJ, USA) following digestion with the suitable restriction endonuclease and ligation by T4 DNA ligase (Promega), resulting in the pUL142-GFP plasmid.

The Snapin coding sequence from pACT2-Snapin was cloned into the pDsRed-C1 vector (BD Biosciences) with the EcoRI (introduced by the forward primer, 5'-CCGGAA TTCTGCGGGGGCTGGTTCCGCCGC-3') and KpnI (by the reverse primer, 5'-CGGGGTACCTTATTTGCCTGG GGAGCCA-3') sites, to produce the gene-rating plasmid pDsRed-Snapin. All constructs were confirmed by sequencing and sequence analysis (Invitrogen Life Technologies).

HEK-293 cells at $75 \%$ confluence were co-transfected with $1 \mu \mathrm{g}$ pUL142-GFP and $1 \mu \mathrm{g}$ pDsRed-Snapin using X-tremeGENE HP DNA Transfection Reagent (Roche, Mannheim, Germany) for a $24 \mathrm{~h}$ transfection period, which was subsequently replaced by normal growth media. The cells were analyzed using a TCS SP2 Nikon laser scanning confocal microscope (Nikon Eclipse C1 Plus, Tokyo, Japan) with 488-nm and 543-nm excitation beams at $48 \mathrm{~h}$ post-transfection.

\section{Results}

Snapin was screened as a binding protein of HCMV pUL142 with a yeast two-hybrid assay. Putative pUL142 binding proteins were identified from a human fetal brain cDNA library by yeast two-hybrid assay. Results of autonomous activation tests carried out using the UL142-BD fusion protein (pGBKT7-UL142) showed no autoactivation was caused by pUL142 in this system.

Among the positive clones identified from the cDNA library, four were confirmed by sequencing to contain the cDNA sequences of Snapin. Two of these contained the complete coding sequence of Snapin with more than $99 \%$ nucleotide identity. Yeast cells expressing AD-Snapin and BD-UL142 fusion proteins grew well on the SD medium lacking Ade/Trp/Leu/His, and turned blue in a further chromogenic reaction. These results strongly indicated that pUL142 binds to the Snapin protein in yeast.

Interaction between HCMV pUL142 and Snapin in vitro was confirmed by GST pull-down assay. GST pull-down 

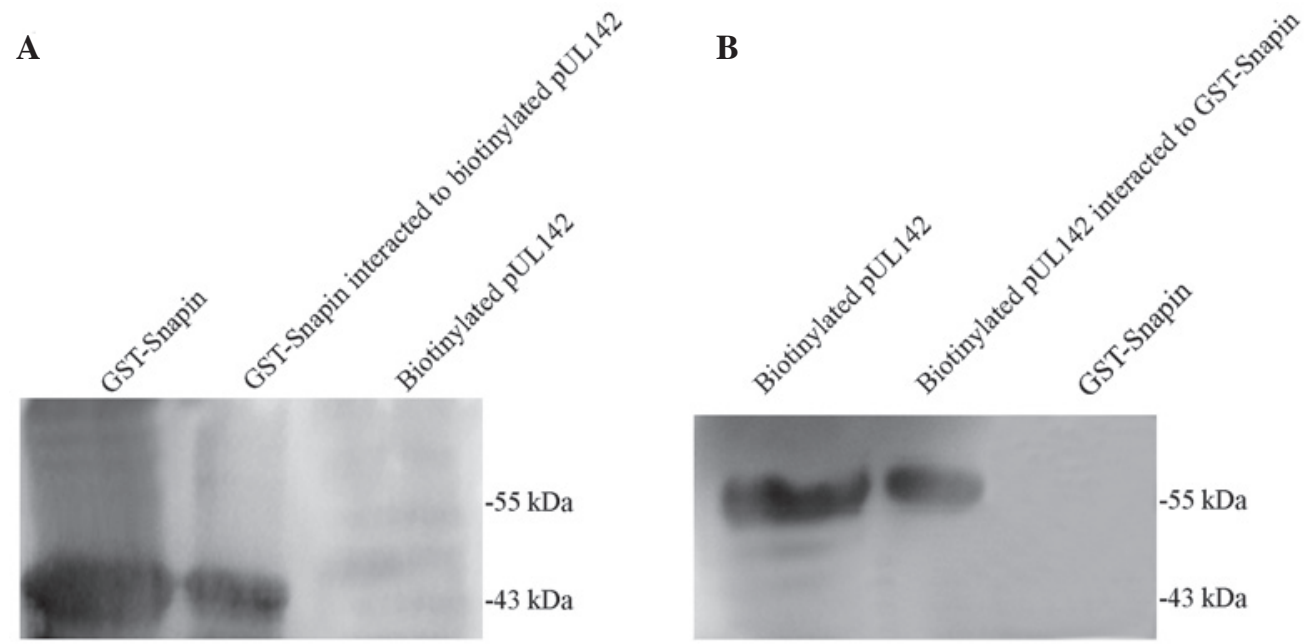

Figure 1. Results of pull-down experiments analyzed by western blotting. (A) GST-Snapin detected with a mouse anti-GST monoclonal antibody. Lane 1: GST-Snapin expressed in transformed BL21. Lane 2: GST-Snapin and pUL142 captured by MagneGST ${ }^{\mathrm{TM}}$ particles. Lane 3: the UL142 protein alone. (B) Biotinylated pUL142 was detected with streptavidin and streptavidin-horseradish peroxidase. Lane 1: the UL142 protein expressed in the TNT system. Lane 2: the biotinylated pUL142 protein bound to GST-Snapin, which was captured by MagneGST ${ }^{\mathrm{TM}}$ particles. Lane 3: GST-Snapin alone. GST, glutathione S transferase; pUL142, UL142-encoded protein.

A

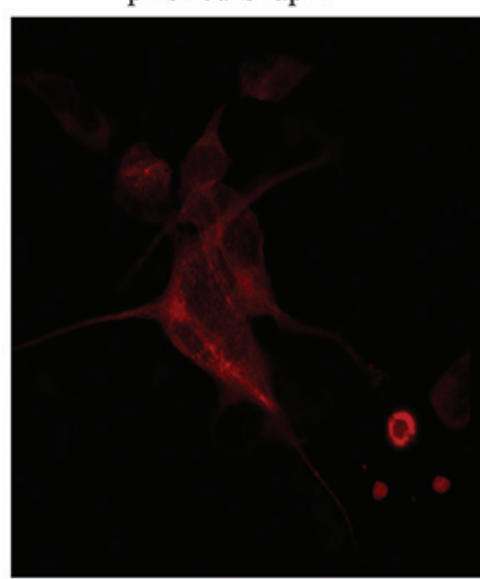

B

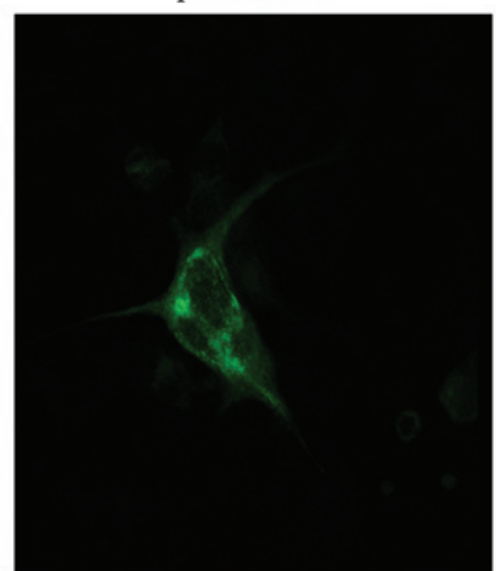

C

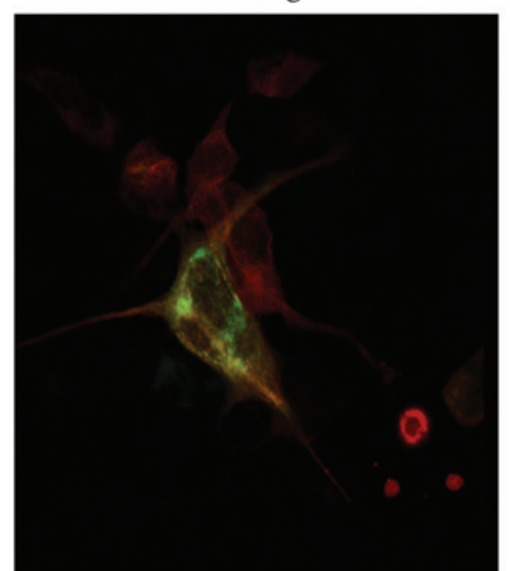

Figure 2. Immunofluorescent tests. (A) The fusion protein expressed by pDsRed-Snapin was localized in the cytoplasm, as was the (B) pUL142-GFP fusion protein. (C) Confocal microscopic analysis revealed that pUL142 fusion protein co-localized with Snapin fusion protein in the cytoplasm in human embryonic kidney-293 cells. UL142-encoded protein, pUL142; GFP, green fluorescent protein.

experiments were performed to verify the direct interaction between pUL142 and Snapin. Consistent with the yeast two-hybrid assay results, pUL142 was specifically bound to GST-Snapin. As shown in Fig. 1, GST-Snapin (Fig. 1A, lane 1) and biotinylated pUL142 (Fig. 1B, lane 1) were highly expressed in the transformed BL21 and the TNT system, respectively. Following the incubation of the two expressed proteins with MagneGST ${ }^{\mathrm{TM}}$ particles, GST-Snapin and pUL142 were detected in the recovered proteins (Fig. 1A, lane 2 and Fig. 1B, lane 2, respectively). In contrast, no corresponding band was observed in the control lanes (Fig. 1A, lane 3, and Fig. 1B, lane 3). This result confirmed that pUL142 was able to be captured by GST-Snapin, and that there was a direct interation between Snapin and pUL142 in this in vitro experiment.

Colocalization of HCMV pUL142 and Snapin was detected by immunofluorescence assay in the HEK-293 cells. To detect whether pUL142 had the same localization as that of Snapin, the plasmids pUL142-GFP and pDsRed-Snapin were co-transfected into HEK-293 cells and their expressed proteins were detected by immunofluorescence assay. As shown in Fig. 2, the fusion proteins expressed by pDsRed-Snapin and pUL142-GFP were broadly expressed and spatially colocalized in the transfected HEK-293 cells, indicating that they could form a complex in the transient expression system.

\section{Discussion}

Interactions between virus and host proteins may be an important method for viruses to establish a suitable environment for replication and dissemination. Understanding the potential interactions between viral proteins and those between viral and human proteins is important for elucidating the mechanisms of infection and developing novel strategies for the treatment 
and prevention of herpesvirus latency and infection (14-16). As a novel HCMV encoded major histocompatibility complex (MHC) class I-related molecule, the UL142 protein contains an MHC class I Antigen (MICA) recognition domain $(17,18)$, which is able to downregulate the expression levels of the NKG2D ligand, leading to protection from NK cytotoxicity and inhibition of NK cell-mediated lysis (19-22).

Snapin, expressed in a number of types of cells, including adipocytes and neuronal cells (23-26), has been established to be associated with the soluble $\mathrm{N}$-ethylmaleimide-sensitive factor attachment protein receptor (SNARE) complex $(27,28)$. SNAREs are ubiquitous proteins that direct vesicular trafficking and exocytosis, and mediate the release of neurotransmitters in neurons (29). Zhou et al (30), have revealed that Snapin has a critical role in coordinating dynein-driven retrograde transport and late endosomal-lysosomal trafficking, thus maintaining efficient autophagy-lysosomal function. In addition, a number of studies have demonstrated that Snapin may regulate HCMV genomic DNA synthesis by modulating the cellular distribution of viral helicase $(25,31-33)$.

In the present study, a direct interaction between pUL142 and Snapin was confirmed in vitro. Furthermore, Snapin and pUL142 were demonstrated to be highly colocalized in cotransfected HEK-293 cells. These results indicate that the interaction of pUL142 with the host protein Snapin may occur in vivo and influence vesicular trafficking and exocytosis by increasing the formation of the virus releasable pool and synaptic transmission. However, the biological functions of the interaction between pUL142 and Snapin in vivo are still in question and details of this aspect require further investigation.

\section{Acknowledgements}

This study was supported by grants from the National Natural Science Foundation of China (nos. 30672248, 81171580, 81171581, 81201274 and 81371788) and the Specialized Research Fund for the Doctoral Program of Higher Education (no.: 20112104110012) and the Outstanding Scientific Fund of Shengjing Hospital and the Natural Science Foundation of Liaoning Province, China (no. 201202283).

\section{References}

1. Malm G and Engman ML: Congenital cytomegalovirus infections. Semin Fetal Neonatal Med 12: 154-159, 2007.

2. Fishman JA and Rubin RH: Infection in organ-transplant recipients. New Engl J Med 338: 1741-1751, 1998.

3. Alford CA, Stagno S, Pass RF and Britt WJ: Congenital and perinatal cytomegalovirus infections. Rev Infect Dis 12: 745-753, 1990.

4. Dolan A, Cunningham C, Hector RD, et al: Genetic content of wild-type human cytomegalovirus. J Gen Virol 85: 1301-1312, 2004.

5. Murphy E, Yu D, Grimwood J, et al: Coding potential of laboratory and clinical strains of human cytomegalovirus. Proc Natl Acad Sci USA 100: 14976-14981, 2003.

6. CheeMS,Bankier AT, BeckS, et al: Analysis of the protein-coding content of the sequence of human cytomegalovirus strain AD169. Curr Top Microbiol Immunol 154: 125-169, 1990.

7. Davison AJ, Dolan A, Akter P, et al: The human cytomegalovirus genome revisited: comparison with the chimpanzee cytomegalovirus genome. J Gen Virol 84: 17-28, 2003.

8. Cha TA, Tom E, Kemble GW, Duke GM, Mocarski ES and Spaete RR: Human cytomegalovirus clinical isolates carry at least 19 genes not found in laboratory strains. J Virol 70: 78-83, 1996.

9. Varnum SM, Streblow DN, Monroe ME, et al: Identification of proteins in human cytomegalovirus (HCMV) particles: the HCMV proteome. J Virol 78: 10960-10966, 2004.
10. Grainger L, Cicchini L, Rak M, Petrucelli A, Fitzgerald KD, Semler BL and Goodrum F: Stress-inducible alternative translation initiation of human cytomegalovirus latency protein pUL138. J Virol 84: 9472-9486, 2010.

11. Umashankar M, Petrucelli A, Cicchini L, et al: A novel human cytomegalovirus locus modulates cell type-specific outcomes of infection. PLoS Pathog 7: e1002444, 2011.

12. Wang YP, Qi Y, Huang YJ, et al: Identification of immediate early gene X-1 as a cellular target gene of hcmv-mir-UL148D. Int J Mol Med 31: 959-966, 2013.

13. Wills MR, Ashiru O, Reeves MB, et al: Human cytomegalovirus encodes an MHC class I-like molecule (UL142) that functions to inhibit NK cell lysis. J Immunol 175: 7457-7465, 2005.

14. Pei Y, Fu W, Yang E, et al: A Hsp40 chaperone protein interacts with and modulates the cellular distribution of the primase protein of human cytomegalovirus. PLoS Pathog 8: e1002968, 2012.

15. Paulus C, Krauss S and Nevels M: A human cytomegalovirus antagonist of type I IFN-dependent signal transducer and activator of transcription signaling. Proc Natl Acad Sci USA 103: 3840-3845, 2006.

16. Cristea IM, Moorman NJ, Terhune SS, et al: Human cytomegalovirus pUL83 stimulates activity of the viral immediate-early promoter through its interaction with the cellular IFI16 protein. J Virol 84: 7803-7814, 2010.

17. Bauer S, Groh V, Wu J, Steinle A, Phillips JH, Lanier LL and Spies T: Activation of NK cells and T cells by NKG2D, a receptor for stress-inducible MICA. Science 285: 727-729, 1999.

18. Wu J, Song Y, Bakker AB, Bauer S, Spies T, Lanier LL and Phillips JH: An activating immunoreceptor complex formed by NKG2D and DAP10. Science 285: 730-732, 1999.

19. Chalupny NJ, Rein-Weston A, Dosch S and Cosman D: Down-regulation of the NKG2D ligand MICA by the human cytomegalovirus glycoprotein UL142. Biochem Biophys Res Commun 346: 175-181, 2006.

20. Zou Y, Bresnahan W, Taylor RT and Stastny P: Effect of human cytomegalovirus on expression of MHC class I-related chains A. J Immunol 174: 3098-3104, 2005.

21. Beck S and Barrell BG: Human cytomegalovirus encodes a glycoprotein homologous to MHC class I antigens. Nature 331: 269-272, 1988.

22. Novotny J, Rigoutsos I, Coleman D and Shenk T: In silico structural and functional analysis of the human cytomegalovirus (HHV5) Genome. J Mol Biol 310: 1151-1166, 2001.

23. Bao Y, Lopez JA, James DE and Hunziker W: Snapin interacts with the Exo70 subunit of the exocyst and modulates GLUT4 trafficking. J Biol Chem 283: 324-331, 2008.

24. Buxton P, Zhang XM, Walsh B, Sriratana A, Schenberg I, Manickam E and Rowe T: Identification and characterization of Snapin as a ubiquitously expressed SNARE-binding protein that interacts with SNAP23 in non-neuronal cells. Biochem J 375: 433-440, 2003.

25. Luo J, Chen J, Yang E, et al: Modulation of the cellular distribution of human cytomegalovirus helicase by cellular factor snapin. J Virol 87: 10628-10640, 2013.

26. Barnard EC, Brown G and Stow ND: Deletion mutants of the herpes simplex virus type 1 UL8 protein: effect on DNA synthesis and ability to interact with and influence the intracellular localization of the UL5 and UL52 proteins. Virology 237: 97-106, 1997.

27. Söllner TH: Regulated exocytosis and SNARE function. Mol Membr Biol 20: 209-220, 2003.

28. Weber T, Zemelman BV, McNew JA, et al: SNAREpins: minimal machinery for membrane fusion. Cell 92: 759-772, 1998.

29. Penfold ME and Mocarski ES: Formation of cytomegalovirus DNA replication compartments defined by localization of viral proteins and DNA synthesis. Virology 239: 46-61, 1997.

30. Zhou B, Zhu YB, Lin L, Cai Q and Sheng ZH: Snapin deficiency is associated with developmental defects of the central nervous system. Biosci Rep 31: 151-158, 2011.

31. Wu CA, Nelson NJ, McGeoch DJ and Challberg MD: Identification of herpes simplex virus type 1 genes required for origin-dependent DNA synthesis. J Virol 62: 435-443, 1988.

32. Suzuki F, Morishima S, Tanaka T and Muramatsu I: Snapin, a new regulator of receptor signaling, augments alpha1 A-adrenocept-or-operatedcalcium influx through TRPC6. J Biol Chem 282: 29563-29573, 2007.

33. Snyder DA, Kelly ML and Woodbury DJ: SNARE complex regulation by phosphorylation. Cell Biochem Biophys 45: 111-123, 2006. 